\title{
Gastrointestinal Adverse Effects of Immunotherapeutic Agents: A Systematic Review
}

\author{
Dushyant Singh Dahiya ${ }^{\text {a, d }}$, Farah Wani ${ }^{\text {b }}$, Jean Claude Guidic ${ }^{\mathrm{c}}$, Asim Kichloo ${ }^{\mathrm{a}, \mathrm{c}}$
}

\begin{abstract}
The utilization of immunotherapy is increasing to the point of becoming the fifth pillar of management alongside surgical intervention, chemotherapy, radiotherapy and targeted therapy. However, gastrointestinal adverse effects and toxicities have been frequently cited with its use. As per literature, the most common adverse effect of immune checkpoint inhibitors is watery and non-bloody diarrhea. Adoptive cell therapy can lead to delayed, on-target but off-tumor adverse effects which are unknown and may be life-threatening. The use of anti-angiogenic monoclonal antibodies can lead to bowel perforations, whereas epidermal growth factor receptor inhibitors and anti-HER2 agents are frequently associated with diarrhea. Minimal adverse effects have been associated with therapeutic cancer vaccines; however, additional studies are needed to determine their efficacy and potential toxicities. To provide an in-depth review of the gastrointestinal side effects of immunotherapeutic agents, we performed a thorough literature search using multiple online search engines such as PubMed, Google Scholar and Ovid MEDLINE, along with a review of the guidelines from the United States Food and Drug Administration (FDA) and the Cancer Research Institute on immunotherapy. In this systematic review, we detail the gastrointestinal adverse effects of immunotherapy and describe their management. With the advent of newer immunotherapeutic agents and the consistent approval of current agents by FDA for a wide spectrum of cancers, it is vital for physicians to familiarize themselves with their adverse effects for prompt diagnosis and early intervention to decrease adverse outcomes.
\end{abstract}

Keywords: Immunotherapy; Gastrointestinal; Adverse effects; Immune checkpoint inhibitors; Adoptive cell therapy; Therapeutic cancer vaccines; Systematic review; Diarrhea; Colitis

Manuscript submitted November 6, 2020, accepted December 5, 2020

Published online December 23, 2020

aDepartment of Internal Medicine, Central Michigan University, Saginaw, MI, USA

bDepartment of Family Medicine, Samaritan Medical Center, Watertown, NY, USA

'Department of Internal Medicine, Samaritan Medical Center, Watertown, NY, USA

${ }^{\mathrm{d} C o r r e s p o n d i n g ~ A u t h o r: ~ D u s h y a n t ~ S i n g h ~ D a h i y a, ~ C e n t r a l ~ M i c h i g a n ~ U n i v e r-~}$ sity College of Medicine, 1000 Houghton Avenue, Saginaw, MI 48602, USA. Email: dush.dahiya@gmail.com

doi: https://doi.org/10.14740/gr1340

\section{Introduction}

In 1909, Smith first speculated that the immune system could supress the growth of carcinomas by recognizing tumor cells as foreign antigens [1]. Hence, the immune system became an area of particular interest to investigators due to its specificity in targeting tumor-specific antigens. Immunotherapy is primarily focused on the development of agents to stimulate or supress the immune system as needed to fight off a wide spectrum of diseases, particularly cancer. Progress in the field is largely based on the identification of new immune based targets and a better understanding of the workings of the immune system [2]. Although still in its infancy stage, cancer immunotherapy (CI) is rapidly evolving and advancing to a point that it is considered a separate therapeutic entity and a potential fifth pillar in the management of cancers alongside surgical intervention, chemotherapy, radiotherapy and targeted therapy [3].

\section{Methods}

A thorough literature review was performed looking for articles related to immunotherapy and cancer. The authors used search engines such as PubMed, Google Scholar and Ovid MEDLINE to look for published articles on immunotherapy between the years 1990 and 2020. A detailed literature search of the articles referenced in the reviewed articles was also performed. Additionally, data and statistics available from professional organizations such as the United States Food and Drug Administration (FDA) and Cancer Research Institute were also thoroughly researched for immunotherapy. The keywords used in the literature search included, but not limited to: "immunotherapy", "cancer", "malignancy", "immune checkpoint inhibitor", "adoptive cell therapy", "monoclonal antibodies", "treatment vaccines", "immune system modulators", "FDA", "adverse effects" and "gastrointestinal". The inclusion criteria included the articles published between the years 1990 and 2020, articles that were available in the English language and articles or guidelines related to the use of different immunotherapeutic agents in all clinical settings. The exclusion criteria consisted of duplicate articles, abstracts only, articles in a language other than English and unpublished research in immunotherapy. The process of literature review is summarized in Figure 1. Application of the inclusion and exclusion criteria yielded a total of 27 articles which were ultimately chosen by 


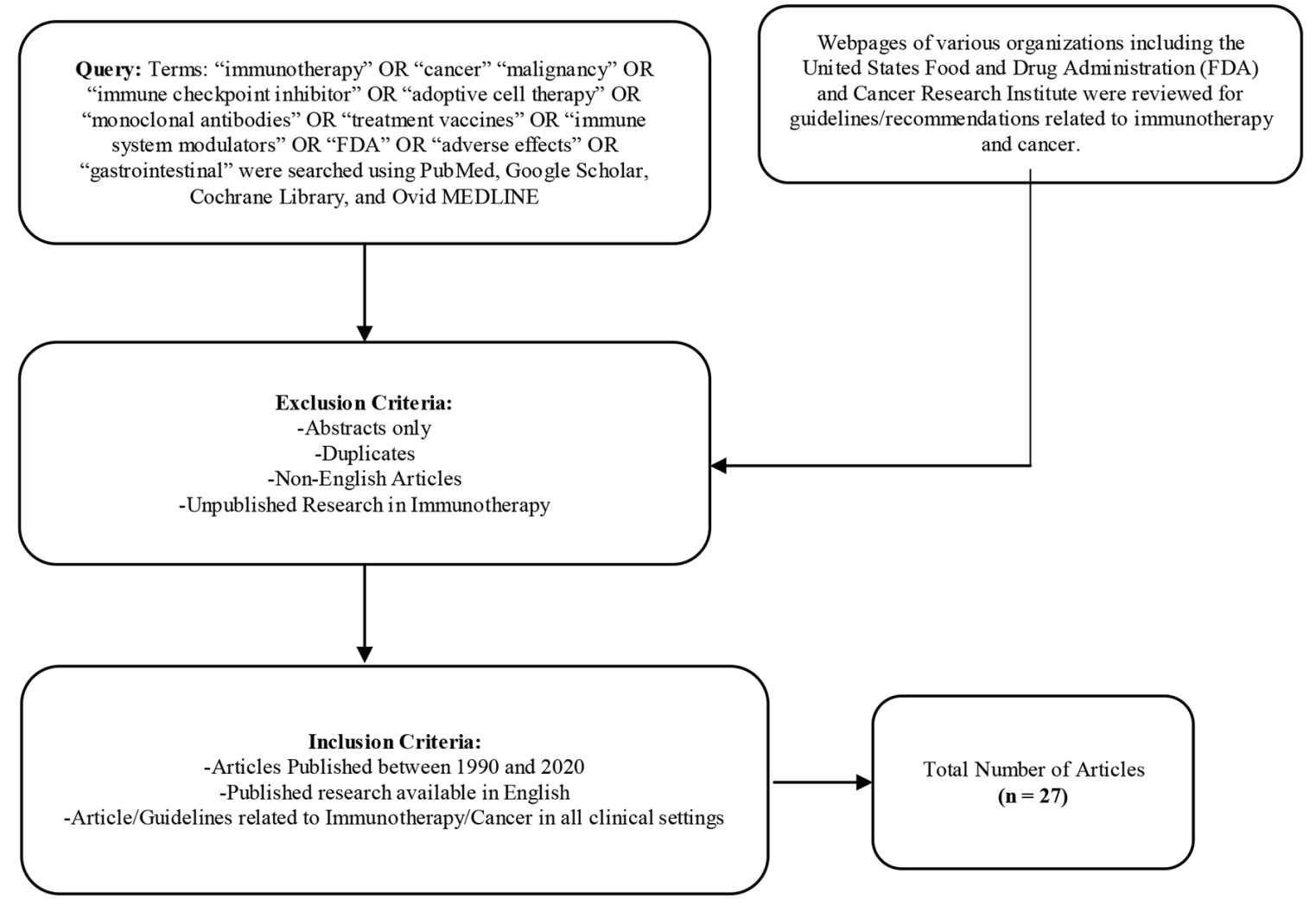

Figure 1. Flowchart outlining process of literature review including inclusion/exclusion criteria.

the authors for this systematic review of literature.

\section{Discussion}

According to the National Cancer Institute (NCI) which is a part of the National Institutes of Health (NIH) and a pioneer in cancer research, the immunotherapeutic agents available for the treatment of cancers can be subdivided into five major subtypes which include [4]: 1) Immune checkpoint inhibitors (ICIs); 2) Adoptive cell therapy; 3) Monoclonal antibodies; 4) Treatment vaccines; and 5) Immune system modulators.

In this section, we will describe the mechanism of action of each of these subtypes and discuss primarily the gastrointestinal (GI) side effect profile and associated toxicity with the use of different agents.

\section{ICIs}

\section{Mechanism of action}

It is a class of antibody that blocks the "immune checkpoint" through the programmed cell death protein-1 (PD-1), programmed death-ligand 1 (PD-L1) or the cytotoxic T-lymphocyte associated protein-4 (CTLA-4) pathways. PD-1 is a surface protein present on T-lymphocytes and acts as an immu- nological "off switch". Through the presentation of the PD-1 receptor with PD-ligand, tumors cells are able to evade recognition for apoptosis and gain the potential of indefinite replication; hence, leading to the development of cancer [5]. CTLA-4 acts in a similar fashion as the immune checkpoint receptor and downregulates the immune responses when bound [6]. ICIs reinvigorate anti-tumor immune response by interrupting the co-inhibitory signaling pathways and promoting immunemediated elimination of the tumor cells [7].

\section{GI side effects}

The side effects associated with ICIs tend to be mild and transient as the agents are generally well tolerated. The mostly frequently reported side effect with the use of ICI is watery, non-bloody diarrhea [8]. It may also present as colitis which is characterized by the presence of bloody diarrhea with fever, abdominal pain, or mucus in the stool. As per literature, a more severe form of diarrhea or colitis may be seen with the use of a combination therapy of anti-CTLA-4 and anti-PD-1 agents rather than with either ipilimumab or anti-PD-1 alone [9]. AntiCTLA-4 colitis may also be associated with mouth ulcers, anal lesions such as fistulas, abscesses or fissures, and other extraintestinal manifestations [10]. Literature also reports other GI side effects associated with the use ICI including abdominal pain, nausea, vomiting, hematochezia and mucus in the stools [11]. Some patients may also have asymptomatic elevations in 
Table 1. The Grading of Diarrhea and Colitis in Patients on Immune Checkpoint Inhibitor Therapy [14]

\begin{tabular}{|c|c|c|c|c|c|}
\hline & Grade 1 & Grade 2 & Grade 3 & Grade 4 & Grade 5 \\
\hline Diarrhea & $\begin{array}{l}\text { Increase of }<4 \text { stools } \\
\text { per day over baseline or } \\
\text { mild increase in ostomy } \\
\text { output versus baseline. }\end{array}$ & $\begin{array}{l}\text { Increase of } 4-6 \text { stools } \\
\text { per day over baseline or } \\
\text { moderate increase in ostomy } \\
\text { output versus baseline. }\end{array}$ & $\begin{array}{l}\text { Increase of } \geq 7 \text { stools per day } \\
\text { over baseline or severe increase } \\
\text { in ostomy output versus baseline. } \\
\text { Hospitalization indicated. }\end{array}$ & $\begin{array}{l}\text { Life-threatening } \\
\text { consequences. } \\
\text { Urgent intervention } \\
\text { indicated. }\end{array}$ & Death \\
\hline Colitis & $\begin{array}{l}\text { Asymptomatic. } \\
\text { Clinical or diagnostic } \\
\text { observations only. } \\
\text { Intervention not indicated. }\end{array}$ & $\begin{array}{l}\text { Abdominal pain; mucus } \\
\text { or blood in stool. }\end{array}$ & $\begin{array}{l}\text { Severe abdominal pain; } \\
\text { peritoneal signs. }\end{array}$ & $\begin{array}{l}\text { Life-threatening } \\
\text { consequences. } \\
\text { Urgent intervention } \\
\text { indicated. }\end{array}$ & Death \\
\hline
\end{tabular}

the liver function tests [12]. Although the upper GI tract is not commonly involved with the use of ICIs, some reported side effects include gastritis, esophagitis and aphthous ulcers [9].

In patients presenting to the hospital with ICI-induced colitis, other differential diagnosis such as GI infections or symptoms related to the underlying cancer should always be ruled out. A stool analysis from these patients can be used to rule out a possible infection secondary to bacterial entero-pathogens, parasites or Clostridium difficile [13]. In some cases, additional imaging modalities such as a computer tomography (CT) scan may be used to differentiate the presenting symptoms from an underlying malignancy. Patients with inflammatory bowel disease (IBD) may present with a similar clinical picture as ICIinduced colitis; hence, clinical correlation of the presenting symptoms, a high degree of clinical suspicion and a thorough medication history are often vital to differentiate the two [8]. Furthermore, in patients with disseminated melanoma, GI metastasis should be ruled out. The gold standard test to establish a definitive diagnosis of ICI-induced colitis is endoscopic evaluation with biopsy followed by a histopathological analysis of the specimen [13]. The grades of diarrhea and colitis are summarized in Table 1 [14]. The management of GI toxicity with the use of ICIs is based on the degree of severity and can be summarized as follows [14, 15]. 1) Mild diarrhea/colitis (grade 1 ): The management is usually supportive with antidiarrheals, fluids and electrolyte replacement. ICI therapy can be continued. 2) Moderate diarrhea/colitis (grade 2): The management includes administration of antidiarrheals, fluids and electrolytes, along with prompt initiation of 0.5 to $1 \mathrm{mg} / \mathrm{kg} /$ day of oral corticosteroids. ICI therapy should be withheld. Gastroenterology specialists should be consulted to confirm the diagnosis with endoscopy and histopathology. Thromboprophylaxis with low molecular weight heparin (LMWH) should be considered as these patients are at increased risk. If the patient does not show any improvement with oral corticosteroids, intravenous (IV) corticosteroids should be considered. 3) Severe diarrhea/ colitis (grade 3 or 4): The patient should be hospitalized with immediate initiation of IV corticosteroids $1-2 \mathrm{mg} / \mathrm{kg}$ per day. ICI therapy should be withheld. If the patient responds well to IV corticosteroids within 3 - 5 days, it should be switched to oral and tapered over $8-12$ weeks. If the patient does not improve with IV steroid therapy within 3 - 5 days or has a relapse during the steroid taper, a rapid escalation to infliximab $5 \mathrm{mg}$ / $\mathrm{kg}$ is recommended. If there is a suboptimal response to $5 \mathrm{mg} /$ $\mathrm{kg}$ infliximab, a higher dose of $10 \mathrm{mg} / \mathrm{kg}$ can be considered. Usually, patients respond well to the single dose of infliximab; however, some may need a second dose about 2 weeks later. Vedolizumab, a monoclonal antibody, could potentially also be considered for steroid-refractory or steroid-dependent colitis.

Severe life-threatening enterocolitis refractory to therapy and progressing to bowel perforation requires surgical intervention with colectomy with ileostomy. Restarting the patients on immunotherapy after an episode of enterocolitis should be individualized to the patient and requires risk-benefit assessment. However, in patients with severe diarrhea or colitis, it is recommended that immunotherapy be permanently discontinued.

\section{T-cell transfer therapy (adoptive cell therapy)}

\section{Mechanism of action}

Adoptive T-cell transfer is a form of passive immunization which can be achieved through [16]: 1) Collection of activated $\mathrm{T}$ cells from the cancer tissue, stimulation with interleukin-2 (IL-2) in vitro and then infusion of the cells back into the patient. 2) Genetically engineering $T$ cells, translocating chimeric antigen receptor (CAR) T cells or transducing the antigenspecific T-cell receptor (TCR) into T (TCR-T) cells.

The main goal of T-cell transfer therapy is the creation of tumor-specific $T$ cells that can recognize and eliminate cancerous cells through specific mechanisms.

\section{GI side effects}

The infusion of T cells is generally well tolerated by most patients. Infusion-related events, although infrequent, are usually mild and mostly commonly secondary to the cryoprotectant, dimethyl sulphoxide, or concomitant medication [17]. Literature reports that T-cell therapy may also be associated with on-target adverse effects which are usually not life-threatening, but may limit the treatment strategy. These on-target adverse effects are expected as the engineered T cells may share specific target antigens with different organ systems. Some of the common GI specific on-target adverse effects include [18]: 1) T-cell therapy for colorectal cancer: leading to colitis or acute pulmonary infiltrates in a subgroup of patients. It is treated with systemic corticosteroids. 2) T-cell therapy for esophageal cancer: leading to seizures, coma or even death in a subgroup of patients. It can be treated with high dose systemic corticosteroids and anti-epileptic agents. 
As T-cell therapy becomes more potent and effective with a better understanding of the workings of the immune system, acute toxicities may start to become even more apparent. $\mathrm{Cy}$ tokine syndrome, an acute complication of T-cell therapy, is characterized by large scale activation of $\mathrm{T}$ cells upon recognition of the malignant cells and can present with symptoms such as fevers, rigors, hypotension and hypoxia [18].

However, the main concern of T-cell therapy is the potential for delayed, on-target but off-tumor adverse effects, which are currently unknown and may be life-threatening. Current literature does report a case of a patient with colorectal cancer and metastasis to the lungs and liver who was treated with HER2specific CAR T-cell infusion, and eventually passed away 5 days after the initiation of therapy due to multiorgan failure [12]. It is believed that this may have been due to the recognition of HER2 expressed by normal lung tissues leading to a cytokine storm and eventual multiorgan failure. This was an unforeseen event as it has not been observed in HER2 vaccine trials or in patients with breast cancer treated with the HER 2 monoclonal antibodies [18]. Hence, it is recommended that therapy with T cells should always be individualized to the patient, and a through risk-benefit assessment should be performed.

\section{Monoclonal antibodies}

\section{Mechanism of action}

Monoclonal antibodies are engineered to be antigen-specific, often tumor-specific, and mediate the destruction of tumor cells through one of the following mechanisms [19]: 1) Direct tumor cell killing: blockade or agonist activity, induction of apoptosis, or delivery of a drug, radiation, or cytotoxic agent to the tumor cell. 2) Immune-mediated tumor cell killing: phagocytosis, compliment activation, antibody-dependent cell-mediated toxicity (ADCC), or through activated/modified T cells. 3) Vascular and stromal ablation: vessel receptor antagonism, ligand trapping, or stromal cell inhibition.

\section{GI side effects}

The use of monoclonal antibodies has increased significantly over the last decade with the FDA approving more agents for greater number of cancers. The decision to start or discontinue therapy with monoclonal bodies is always patient centric, requires a risk-benefit analysis and depends greatly on the severity of symptoms.

1) Anti-angiogenic agents: These agents (bevacizumab, aflibercept, ramucirumab) are anti-angiogenic and hence, play a critical role in the prevention of tumor growth and spread. Literature reports that bevacizumab has been associated with GI perforations in a dose-dependent fashion with an incidence rate of $1.1 \%$ and a bevacizumab-associated GI perforationrelated mortality of $8.8 \%$ [20]. A similar rate of GI perforation has been noted with aflibercept with a mortality of 10.8\% [21]. The possible mechanism of GI perforations and infarction may be secondary to limitation of blood flow to the GI tract. If a perforation is detected, prompt surgical intervention is necessary along with bowel rest, fluid resuscitation and initiation of broad-spectrum IV antibiotics.

2) Epidermal growth factor receptor (EGFR) inhibitors: These agents (cetuximab, panitumumab) bind to the EGFR and inhibit the downstream receptor signaling. In 2015, the results from a meta-analysis of 18 studies reported that patients had a $66 \%$ increased risk of developing grade 3 - 4 diarrhea while on treatment with cetuximab or panitumumab in combination with chemotherapy compared to chemotherapy alone [22]. Additionally, another common side effect of EGFR inhibitor therapy is hypomagnesemia, which could possibly be the result of chronic diarrhea and is strongly associated with the duration of therapy [23]. In patients on EGFR inhibitors, management of the diarrhea consists of bowel rest, hydration, electrolyte repletion and the use of anti-motility agents. In cases of severe dehydration, hospitalization may become necessary.

3) Anti-HER2 agents: Trastuzumab, a pioneer agent, has been approved by the FDA for the treatment of Her2/neu amplified metastatic gastric or gastroesophageal junction (GEJ) adenocarcinoma. As per the results of the ToGa trial, trastuzumab plus chemotherapy was associated with a higher prevalence of diarrhea compared to chemotherapy alone [24].

\section{Therapeutic cancer vaccines}

\section{Mechanism of action}

Effective cancer vaccines deliver concentrated antigen to both human leukocyte antigen (HLA) class I and II molecules of antigen presenting cells (APCs), promoting both a CD4 and CD8 T-cell response which helps in the generation of an anti-tumor response [25]. Examples of therapeutic cancer vaccines include [26]: 1) Sipuleucel-T (Provenge): it is used for the treatment of advanced prostate cancer. 2) Talimogene laherparepvec (T-VEC): it is used for the treatment of advanced melanoma.

\section{GI side effects}

In contrast to other cytotoxic therapies, cancer vaccines have reported minimal toxicities in a majority of the clinical trials [27]. Despite the presence of multiple target tumor-associated antigens on normal tissues, minimal evidence of autoimmunity has been observed so far with the use of these vaccines [27]. However, their efficacy in the treatment of cancers is yet to be thoroughly evaluated. Most therapeutic cancer vaccines are currently being evaluated in clinical trials.

\section{Conclusion}

Immunotherapy has proven itself to be a worthwhile addition to the arsenal of therapeutic treatment modalities that care providers have against cancers. With the FDA approving more agents for a wide variety of cancers, it is essential that physi- 
cians familiarize themselves with the potential adverse effects and toxicities associated with these agents and their role in cancer treatment strategies. The most reported adverse effect of ICI is watery and non-bloody diarrhea. T-cell therapy could potentially lead to delayed, on-target but off-tumor adverse effects, which are mainly unknown but may be life-threatening. Antiangiogenic monoclonal antibodies are commonly associated with bowel perforations, whereas EGFR inhibitors and antiHER2 agents are frequently associated with diarrhea. Minimal toxicities have been reported with the use of therapeutic cancer vaccines. Thorough patient education along with a risk-benefit assessment, and joint decision making by the provider and the patient will hopefully result in a significant reduction of adverse outcomes. Additionally, it is worth noting that immunotherapy is a relatively newer treatment modality; hence, a significant proportion of adverse effects may be unknown. With a better understanding of the workings of the immune system and future development of highly specific immunotherapy agents, the ratio of benefits to risks may continue to rise.

\section{Acknowledgments}

None to declare.

\section{Financial Disclosure}

The authors have received no funding with respect to research, authorship, and/or publication of this article.

\section{Conflict of Interest}

The authors declare no potential conflict of interest with respect to the research, authorship, and/or publication of this article.

\section{Author Contributions}

All authors have contributed to the manuscript and agree with the final version of the manuscript. The final authorship contribution statement is as below. Dushyant Singh Dahiya and Farah Wani are credited with substantial contribution to the design of the work, literature review of all the sections discussed, the revision of critically important intellectual content, final approval of the published version, and agreement of accountability for all aspects of the work. Jean Claude Guidi and Asim Kichloo are credited with significant design of the tables and graphs, literature review of all sections, revision of important intellectual content for the discussion, and agreement of accountability for all parts of the work.

\section{Data Availability}

The data supporting this systematic review are from previ- ously reported studies and data sets, which have been cited here within.

\section{Abbreviations}

FDA: United States Food and Drug Administration; CI: cancer immunotherapy; NCI: National Cancer Institute; NIH: National Institutes of Health; ICIs: immune checkpoint inhibitors; GI: gastrointestinal; PD-1: programmed cell death protein-1; PD-L1: programmed death-ligand 1; CTLA-4: cytotoxic Tlymphocyte associated protein-4; CT: computed tomography; IBD: inflammatory bowel disease; LMWH: low molecular weight heparin; IV: intravenous; CAR: chimeric antigen receptor; TCR: T-cell receptor; ADCC: antibody-dependent cellmediated toxicity; EGFR: epidermal growth factor receptor; GEJ: gastroesophageal junction; APC: antigen presenting cell; T-VEC: talimogene laherparepvec

\section{References}

1. Smith T. Active immunity produced by so called balanced or neutral mixtures of diphtheria toxin and antitoxin. J Exp Med. 1909;11(2):241-256.

2. Yaguchi T, Kawakami Y. Cancer-induced heterogeneous immunosuppressive tumor microenvironments and their personalized modulation. Int Immunol. 2016;28(8):393399.

3. Oiseth SJ, Aziz MS. Cancer immunotherapy: a brief review of the history, possibilities, and challenges ahead. J Cancer Metastasis and Treat. 2017;3:250-261.

4. Cancer Immunotherapy. Available from: https://www. cancer.gov/about-cancer/treatment/types/immunotherapy. Accessed on: October 15th, 2020.

5. Immune checkpoint inhibitors and their side effects. Available from: https://www.cancer.org/treatment/treatmentsand-side-effects/treatment-types/immunotherapy/immunecheckpoint-inhibitors.html. Accessed on October 15th 2020.

6. Syn NL, Teng MWL, Mok TSK, Soo RA. De-novo and acquired resistance to immune checkpoint targeting. Lancet Oncol. 2017;18(12):e731-e741.

7. Darvin P, Toor SM, Sasidharan Nair V, Elkord E. Immune checkpoint inhibitors: recent progress and potential biomarkers. Exp Mol Med. 2018;50(12):1-11.

8. Assarzadegan N, Montgomery E, Anders RA. Immune checkpoint inhibitor colitis: the flip side of the wonder drugs. Virchows Arch. 2018;472(1):125-133.

9. Marin-Acevedo JA, Harris DM, Burton MC. Immunotherapy-induced colitis: an emerging problem for the hospitalist. J Hosp Med. 2018;13(6):413-418.

10. Marthey L, Mateus C, Mussini C, Nachury M, Nancey S, Grange F, Zallot C, et al. Cancer immunotherapy with Anti-CTLA-4 monoclonal antibodies induces an inflammatory bowel disease. J Crohns Colitis. 2016;10(4):395-401.

11. Puzanov I, Diab A, Abdallah K, Bingham CO, 3rd, Brogdon C, Dadu R, Hamad L, et al. Managing toxicities as- 
sociated with immune checkpoint inhibitors: consensus recommendations from the Society for Immunotherapy of Cancer (SITC) Toxicity Management Working Group. J Immunother Cancer. 2017;5(1):95.

12. Morgan RA, Yang JC, Kitano M, Dudley ME, Laurencot CM, Rosenberg SA. Case report of a serious adverse event following the administration of $\mathrm{T}$ cells transduced with a chimeric antigen receptor recognizing ERBB2. Mol Ther. 2010;18(4):843-851.

13. Haanen J, Carbonnel F, Robert C, Kerr KM, Peters $\mathrm{S}$, Larkin J, Jordan K, et al. Management of toxicities from immunotherapy: ESMO Clinical Practice Guidelines for diagnosis, treatment and follow-up. Ann Oncol. 2017;28(suppl_4):iv119-iv142.

14. Rocha M, Correia de Sousa J, Salgado M, Araujo A, Pedroto I. Management of gastrointestinal toxicity from immune checkpoint inhibitor. GE Port J Gastroenterol. 2019;26(4):268-274.

15. Samaan MA, Pavlidis P, Papa S, Powell N, Irving PM. Gastrointestinal toxicity of immune checkpoint inhibitors: from mechanisms to management. Nat Rev Gastroenterol Hepatol. 2018;15(4):222-234.

16. Zhao Q, Yu J, Meng X. A good start of immunotherapy in esophageal cancer. Cancer Med. 2019;8(10):4519-4526.

17. Cruz CR, Hanley PJ, Liu H, Torrano V, Lin YF, Arce JA, Gottschalk S, et al. Adverse events following infusion of T cells for adoptive immunotherapy: a 10-year experience. Cytotherapy. 2010;12(6):743-749.

18. Tey SK. Adoptive T-cell therapy: adverse events and safety switches. Clin Transl Immunology. 2014;3(6):e17.

19. Scott AM, Allison JP, Wolchok JD. Monoclonal antibodies in cancer therapy. Cancer Immun. 2012;12:14.

20. Qi WX, Shen Z, Tang LN, Yao Y. Bevacizumab increases the risk of gastrointestinal perforation in cancer patients: a meta-analysis with a focus on different subgroups. Eur J Clin Pharmacol. 2014;70(8):893-906.

21. Qi WX, Shen F, Qing Z, Xiao-Mao G. Risk of gastrointestinal perforation in cancer patients treated with aflibercept: a systematic review and meta-analysis. Tumour Biol. 2014;35(11):10715-10722.

22. Miroddi M, Sterrantino C, Simonelli I, Ciminata G, Phillips RS, Calapai G. Risk of grade 3-4 diarrhea and mucositis in colorectal cancer patients receiving anti-EGFR monoclonal antibodies regimens: A meta-analysis of 18 randomized controlled clinical trials. Crit Rev Oncol Hematol. 2015;96(2):355-371.

23. Arora N, Gupta A, Singh PP. Biological agents in gastrointestinal cancers: adverse effects and their management. J Gastrointest Oncol. 2017;8(3):485-498.

24. Bang YJ, Van Cutsem E, Feyereislova A, Chung HC, Shen L, Sawaki A, Lordick F, et al. Trastuzumab in combination with chemotherapy versus chemotherapy alone for treatment of HER2-positive advanced gastric or gastro-oesophageal junction cancer (ToGA): a phase 3, open-label, randomised controlled trial. Lancet. 2010;376(9742):687-697.

25. Melief CJ, van Hall T, Arens R, Ossendorp F, van der Burg SH. Therapeutic cancer vaccines. J Clin Invest. 2015;125(9):3401-3412.

26. Cancer Vaccines. Available from: https:/www.cancer. org/treatment/treatments-and-side-effects/treatmenttypes/immunotherapy/cancer-vaccines.html. Accessed on October 15th 2020.

27. Guo C, Manjili MH, Subjeck JR, Sarkar D, Fisher PB, Wang XY. Therapeutic cancer vaccines: past, present, and future. Adv Cancer Res. 2013;119:421-475. 